Escoto-Murillo, A., \& Alfaro, E. J. (2021). Análisis de eventos fríos y cálidos por medio de datos de buceo: un enfoque de ciencia ciudadana en el Golfo de Papagayo, Costa Rica. Revista de Biología Tropical, 69(Suppl. 2), S94-S104. https://doi.org/10.15517/rbt.v69iS2.48309

\title{
Análisis de eventos fríos y cálidos por medio de datos de buceo: un enfoque de ciencia ciudadana en el Golfo de Papagayo, Costa Rica
}

\author{
Andrés Escoto-Murillo'; (D) https://orcid.org/0000-0003-4578-2539 \\ Eric J. Alfaro $2,3,4$; (D) https://orcid.org/0000-0001-9278-5017 \\ 1. Programa de Ciencias Marinas, Universidad Nacional, Puntarenas, Costa Rica; pony76.andres@gmail.com \\ 2. Centro de Investigación en Ciencias del Mar y Limnología (CIMAR), Universidad de Costa Rica, San José, Costa \\ Rica; erick.alfaro@ucr.ac.cr \\ 3. Centro de Investigaciones Geofísicas (CIGEFI), Universidad de Costa Rica, San José, Costa Rica. \\ 4. Escuela de Física, Universidad de Costa Rica, San José, Costa Rica.
}

Recibido 29-I-2021. Revisado 01-III-2021. Aceptado 15-IV-2021.

\author{
ABSTRACT \\ Analysis of warm and cool events in the Gulf of Papagayo through diving data: \\ A Citizen Science approach
}

Introduction: In January 2011 and as a Citizen Science initiative, the owners of the Deep Blue Diving Shop in Playa del Coco, Guanacaste, Costa Rica, began to collect daily temperature data in the seawater column during their diving activities, to inform tourists before traveling to Costa Rica and help them properly select their diving equipment and thus maximize the enjoyment of the experience in the Gulf of Papagayo. This data collection remained constant until January 2020 due to the COVID-19 pandemic.

Objective: Identify cold and warm events of the sub-surface temperature of the sea in the Gulf of Papagayo, Guanacaste, Costa Rica and their relationship with known sources of climate variability as El Niño-Southern Oscillation (ENSO) and synoptic systems such as incursions of cold fronts in the Caribbean Sea.

Methods: Sea Subsurface Temperature data corresponding to the lowest temperature of the seawater column were used, at an approximate depth of 25-35m. Observations were made daily, from 01/01/2011 to 01/31/2020. Results: The mean temperature was $25.7^{\circ} \mathrm{C}$. Cooler temperatures were observed in February-March, below $22.5^{\circ} \mathrm{C}$ with a secondary minimum in July. There were two peaks in May and August with temperatures above $27.4^{\circ} \mathrm{C}$. The drops during cold events reached $16-17^{\circ} \mathrm{C}$. All cold events were associated with the passage of cold fronts through the Caribbean Sea, due to reinforcement in the intensity of the trade winds, with a zonal component from the East, which causes seasonal upwelling. The warm events presented temperatures at their maximum of 30-31 ${ }^{\circ} \mathrm{C}$. The latter events were associated with the development and maturity of warm El Niño-type ENSO events. ENSO is an important modulator of sea temperature variability in the Gulf of Papagayo, since El Niño events are related to positive anomalies in sea temperature in the Eastern Tropical Pacific.

Conclusions: The Citizen Science initiative presented in this study proved to be very useful for monitoring sea temperature in the Gulf of Papagayo. The results of this study indicate that Dive Masters can provide data on sea temperature of sufficient quality and with high temporal resolution. Divers can profitably support monitoring and Citizen Science can contribute positively to social well-being by influencing the questions that are being addressed and by giving people a voice in local environmental decision-making. The information generated in this study returns to the tour operators and enhance the understanding of the variability observed in the data collected by them, a knowledge that is later transmitted to their clients to improve their experience.

Key words: Citizen science; Gulf of Papagayo; sea subsurface temperature; upwelling; Central America. 
Los escasos conjuntos de datos oceanográficos y programas de monitoreo a largo plazo hacen que sea fundamental explorar métodos alternativos para la recopilación de datos (Njue et al., 2019). Esto es frecuente en países en desarrollo y de bajos ingresos, donde la escasez de datos es más pronunciada y en donde los métodos de monitoreo convencionales son costosos y desafiantes desde el punto de vista logístico. La Ciencia Ciudadana tiene el potencial de mejorar la creación conjunta de conocimientos y la evidencia científica que sustenta la gobernanza y la gestión de los recursos naturales. Njue et al. (2019) definen la Ciencia Ciudadana como la participación de la ciudadanía en diferentes etapas dentro del proceso de investigación científica, como la recopilación, categorización, transcripción o análisis de datos científicos. Los enfoques de Ciencia Ciudadana difieren en escala, alcance y grado de participación ciudadana. La participación comprende principalmente un modelo de Ciencia Ciudadana contributiva, que involucra a los ciudadanos en la recolección de datos. Njue et al. (2019) presenta numerosos ejemplos de este concepto al campo de la hidrología, incluida Costa Rica (Shahady \& Boniface, 2018).

Según Alfaro et al. (2012), el Golfo de Papagayo se encuentra en una zona donde la variabilidad climática producto del afloramiento costero (diciembre-abril) afecta el comportamiento de las temperaturas superficiales del mar (TSM), las cuales pueden llegar a descender hasta $17{ }^{\circ} \mathrm{C}$ (Alfaro \& Cortés, 2012). Así mismo, durante la época lluviosa (mayo-noviembre) la TSM es más estable ( 28 ${ }^{\circ} \mathrm{C}$ ) (Amador et al., 2006). Este cambio en los patrones de TSM puede tener efectos positivos y negativos sobre la biota presente en este golfo (e.g. Jiménez et al., 2001). Alfaro et al. (2012) encontraron que la variabilidad climática de las temperaturas del mar en el Golfo de Papagayo no solo está influenciada por El Niño-Oscilación del Sur (Alfaro \& Lizano, 2001), en el Pacífico Ecuatorial, sino también por influencias de tipo atmosférico relacionadas con la variabilidad en el Océano Atlántico, debido a que los eventos cálidos (fríos) en
Bahía Culebra, Golfo de Papagayo, tienden a ocurrir en concordancia con anomalías positivas y negativas (negativas y positivas) de los índices Niño 3.4 y OAN, respectivamente.

Sobre la vertiente del Pacífico de Costa Rica la magnitud del viento es normalmente más fuerte durante el invierno y primavera boreal (diciembre a mayo), con dirección predominante del este (vientos alisios), y disminuye durante el verano y otoño boreal, entre junio y noviembre, según explican Alfaro et al. (2012). Durante el invierno-primavera boreal, los frentes fríos que incursionan por el Caribe refuerzan el viento con componente del este sobre el país (Zárate-Hernández, 2013). El viento alisio también puede ser reforzado por la Corriente en Chorro de Bajo Nivel del Caribe (Amador, 2008), que presenta dos máximos, uno en febrero y otro en julio. Alfaro y Cortés (2012) describen que el viento alisio se canaliza entre las tierras bajas del centro de Nicaragua y el norte de Costa Rica. Los vientos producidos por esta canalización son llamados comúnmente como Papagayos o Corriente en Chorro de Papagayo (e.g. Amador et al., 2006), debido al golfo del mismo nombre ubicado en el Pacífico norte de Costa Rica. Estos vientos alisios de magnitud fuerte producen enfriamiento por transferencia de calor latente al aumentar la evaporación, profundizan la capa de mezcla, además de que empujan el agua superficial hacia afuera del golfo, y el agua desplazada es luego reemplazada por agua más fría de las profundidades, presentando un afloramiento costero, especialmente durante el invierno boreal (Alfaro \& Cortés, 2012; Alfaro et al., 2012). Este efecto también ha sido reportado para el sector de Bahía Salinas en un estudio reciente (Alfaro \& Cortés, 2021).

El objetivo de este trabajo es identificar eventos fríos y cálidos de la temperatura subsuperficial del mar (TSbSM) en el Golfo de Papagayo, y su relación con fuentes de variabilidad climática conocidas como El Niño-Oscilación del Sur (ENOS) y sistemas sinópticos como las incursiones de frentes fríos en el Mar Caribe, por medio de los datos recolectados por la empresa de Buceo Deep Blue Diving en 
Playa del Coco, Guanacaste, Costa Rica, entre los años 2011 y 2020. Un segundo objetivo de este trabajo es mostrar cómo esta actividad constituye un ejemplo de Ciencia Ciudadana, el cual es útil para el monitoreo de la variabilidad de la TSbSM en una región de interés científico y de mucha importancia para el sector turismo.

\section{MATERIALES Y MÉTODOS}

Se utilizaron datos de TSbSM recolectados en diferentes sitios de muestreo en el Golfo de Papagayo. La colecta de datos se realizó durante los viajes de buceo recreativo, que diariamente salieron de Playa del Coco, Guanacaste, Costa Rica, a las 8 am en buceo local o hacia el sur a Isla Catalina a las 7:30 am durante todo el año, e incluso hacia el norte a Islas Murciélago (Sector Marino del Parque Nacional Santa Rosa) a las 7:00 am, entre agosto y noviembre. La primera inmersión se realizó a las 8:45 am y la segunda a las 10:30 am, en los mismos puntos de entrada-salida del agua, según protocolos PADI (https://www. padi.com) para equipo SCUBA y mediante sensores SUNTO GECCO. Se registró la temperatura más baja de la columna del agua, a una profundidad máxima aproximada de 25-35 m. Los datos recolectados in situ fueron revisados por los funcionarios de la empresa antes de ser ingresados en su base de datos. En este trabajo, se realizó además una inspección visual para identificar puntos de temperatura atípicos, sin que se encontraran puntos que debieran ser descartados.

Las mediciones se realizaron con una frecuencia casi diaria del 01/01/2011 al $31 / 01 / 2020$, en los diferentes sitios mostrados en la Fig. 1. La ubicación geográfica de estos sitios se detalla en la Tabla 1. Cuando se observó más de un dato al día en los sitios de muestreo, se calculó el promedio de esos datos para ese día en particular. Los días en que no hubo mediciones, se estimaron utilizando el promedio mensual histórico de la serie de tiempo. El porcentaje de datos faltantes del total del registro fue de un $12.6 \%$. Posteriormente, se aplicó una media móvil triangular de 7 datos según lo propuesto por Soley (1994), para filtrar señales de alta frecuencia y así obtener un Índice de Temperatura Subsuperficial del Mar (ITSbSM).

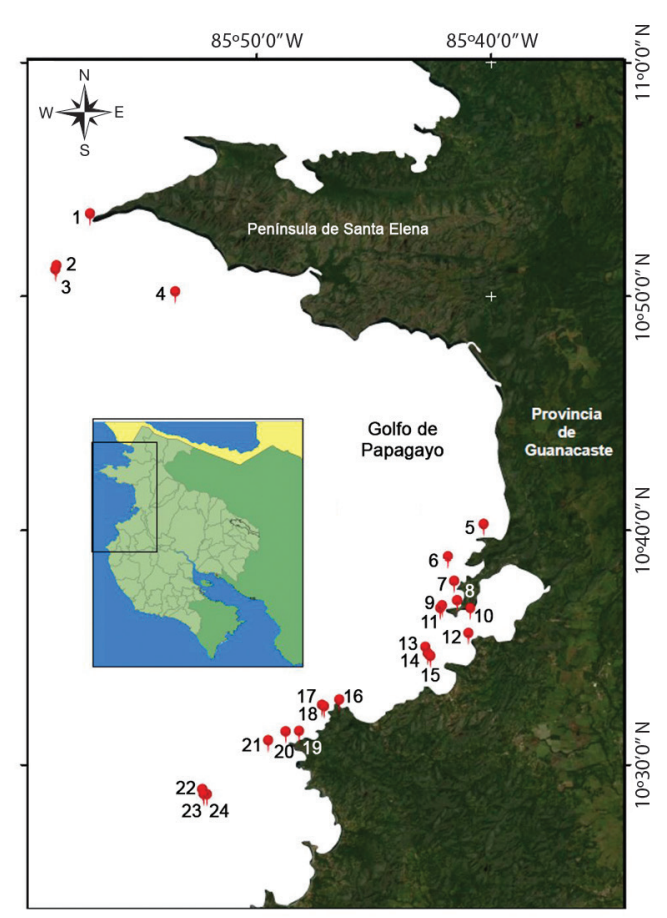

Fig. 1. Ubicación de los de los sitios de muestreo (puntos rojos) en los que se midió la temperatura subsuperficial del mar durante buceos SCUBA en el Pacífico norte de Costa Rica (2011-2020). Su ubicación en grados se muestra en la Tabla 1 .

Fig. 1. Location of the sampling sites (red dots) where the subsurface temperature of the sea was measured during SCUBA dives in the North Pacific of Costa Rica (20112020). Their location in degrees is shown in Table 1.

El registro de los datos de temperatura en la columna de agua de mar surgió como iniciativa en 2011 por parte de los propietarios de la tienda de buceo Deep Blue Diving (http://www.deepblue-diving.com/) en Playa del Coco, como respuesta a la necesidad de informar a los turistas antes de viajar a Costa Rica para la selección del equipo correcto de SCUBA, con el objetivo de maximizar su experiencia y recreación en el Golfo de Papagayo. Este registro se mantuvo constante hasta la pandemia del COVID -19 en enero del año 
2020. La actividad de monitoreo anterior puede considerarse dentro de la definición de Ciencia Ciudadana propuesta por Njue et al. (2019) descrita anteriormente.

TABLA 1

Ubicación geográfica de los sitios de buceo donde se realizaron mediciones de temperatura durante las inmersiones, mostrados como puntos rojos en la Figura 1

TABLE 1

Geographic location of the dive sites where the temperature was measured, shown as red dots in Figure 1

\begin{tabular}{|c|c|c|c|}
\hline Sitio & Nombre & $\begin{array}{l}\text { Latitud } \\
\left({ }^{\circ} \mathrm{N}\right)\end{array}$ & $\begin{array}{c}\text { Longitud } \\
\left({ }^{\circ} \mathrm{W}\right)\end{array}$ \\
\hline 1 & $\operatorname{Arcos}$ & 10.8896276 & -85.952098 \\
\hline 2 & Gran Susto Dos & 10.8529736 & -85.976132 \\
\hline 3 & Gran Susto & 10.8500139 & -85.976803 \\
\hline 4 & Bajo Negro & 10.8344139 & -85.891415 \\
\hline 5 & Mangos & 10.6686401 & -85.671718 \\
\hline 6 & Narizones & 10.6455888 & -85.697014 \\
\hline 7 & Sol y Sombra & 10.6284282 & -85.692636 \\
\hline 8 & Güiri-güiri & 10.6145583 & -85.690503 \\
\hline 9 & Virador & 10.6109786 & -85.701208 \\
\hline 10 & Meros & 10.6091357 & -85.681167 \\
\hline 11 & Cabeza de Mono & 10.6090381 & -85.702562 \\
\hline 12 & Punta Ballena & 10.5913452 & -85.682591 \\
\hline 13 & Sorpresa & 10.5814623 & -85.713127 \\
\hline 14 & Argentina & 10.5771383 & -85.711491 \\
\hline 15 & Tortuga & 10.5751664 & -85.709545 \\
\hline 16 & Punta Gorda & 10.543486 & -85.774495 \\
\hline 17 & Tiburones II & 10.5397016 & -85.786801 \\
\hline 18 & Tiburones & 10.5387272 & -85.785402 \\
\hline 19 & Bonsai & 10.5212514 & -85.803295 \\
\hline 20 & Acuario & 10.5207204 & -85.812776 \\
\hline 21 & Arcoíris & 10.5145703 & -85.825146 \\
\hline 22 & Pared Catalina & 10.4798502 & -85.872106 \\
\hline 23 & Tetas & 10.4764279 & -85.87114 \\
\hline 24 & Punta Sur & 10.4762482 & -85.868974 \\
\hline
\end{tabular}

Además, se consultaron los boletines meteorológicos mensuales (https://www.imn. ac.cr/boletin-meteorologico), elaborados por el Instituto Meteorológico Nacional de Costa Rica (IMN), para analizar las condiciones y sistemas sinópticos a gran escala en los que ocurrieron eventos fríos y cálidos en el Golfo de Papagayo, con el fin de identificar los mecanismos atmosféricos y oceanográficos que favorecieron su ocurrencia. Dicha información se incluye en la descripción de cada uno de los eventos en la próxima sección.

\section{RESULTADOS}

El ciclo anual de los registros de la TSbSM se muestra en la Figura 2. La temperatura media es de $25.7^{\circ} \mathrm{C}$. Se observaron temperaturas más frías en febrero-marzo, por debajo de $22.5{ }^{\circ} \mathrm{C}$ con un mínimo secundario en julio. Hubo dos máximos en mayo y agosto con temperaturas superiores a $27.4{ }^{\circ} \mathrm{C}$. Febrero fue el mes que presentó mayor variabilidad de temperatura y la menor se observó en agosto.

La Fig. 3 muestra el ITSbSM calculado a partir de los registros enumerados en la Tabla 1. Los primeros datos corresponden al 01 de enero de 2011 y los últimos datos corresponden al 31 de enero de 2020. El rango observado del ITSbSM varió de 17.2 a $30.7{ }^{\circ} \mathrm{C}$ para el 8 de marzo de 2013 y el 11 de mayo de 2012, respectivamente. La serie de tiempo no presentó una tendencia significativa a largo plazo (significativa al 95\%) (Fig. 3).

\section{Eventos Fríos}

\section{Evento 1, febrero-marzo, 2013}

Este evento inició el 27/02/2013 y finalizó el 13/03/2013 (Fig. 4), con una duración de 15 días. Su promedio fue de $19.92{ }^{\circ} \mathrm{C}$, con un mínimo de $17.19^{\circ} \mathrm{C}$ el $08 / 03 / 2013\left(14{ }^{\circ} \mathrm{C}\right.$ en el índice sin suavizar).

Según Naranjo (2013) hubo dos empujes fríos en el Mar Caribe, 1-4 marzo y 6-7 marzo de 2013, el primero fue el de mayor afectación para el país. Este llegó hasta Costa Rica, siendo el segundo empuje de la temporada que afectó de forma directa al país, ocasionando abundantes precipitaciones en las regiones del Caribe y la Zona Norte, además de fuertes ráfagas de viento y un descenso de las temperaturas. Naranjo (2013) agrega que, en las imágenes satelitales del 3 de marzo, se observó claramente como el empuje frío abarcó todo el Mar Caribe, afectando a Costa Rica directamente. El análisis de presión atmosférica a nivel de nivel 


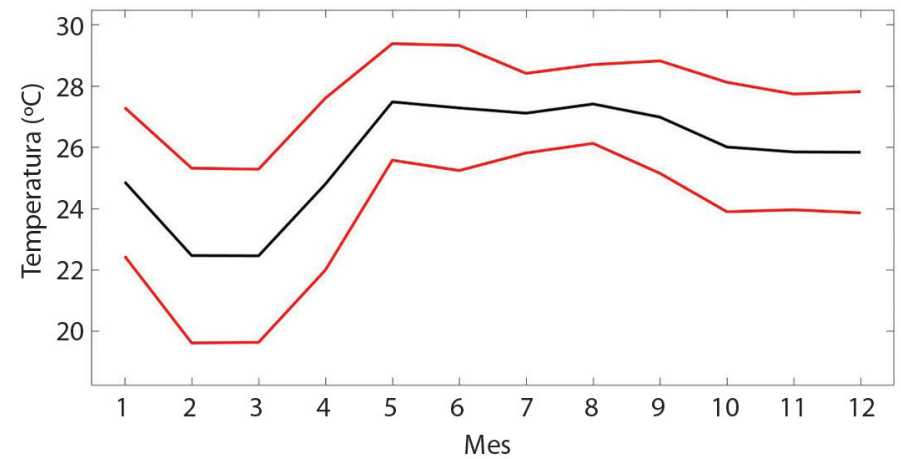

Fig. 2. Temperatura media mensual subsuperficial del mar (TSbSM, línea negra) promedio en el Golfo de Papagayo ( \pm desviación estándar en rojo), a partir de observaciones de ciencia ciudadana tomadas en inmersiones de buceo (2011-2020). Fig. 2. Mean Sea Subsurface Temperature (SSbT, black line) in the Gulf of Papagayo ( \pm standard deviation in red) from the citizen science observations collected during diving activities (2011-2020).

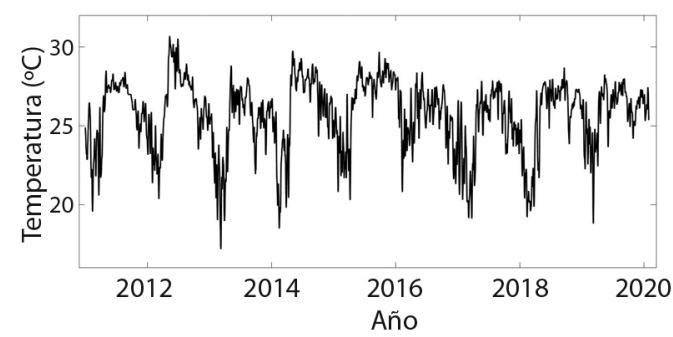

Fig. 3. Índice de Temperatura Subsuperficial del Mar (ITSSM) a partir de los datos de ciencia ciudadana en el Golfo de Papagayo, desde enero de 2011 hasta enero de 2020.

Fig. 3. Sea Subsurface Temperature Index (SSbTI) from the citizen science observations in the Gulf of Papagayo, from January 2011 to January 2020. del mar mostró como el sistema de alta de presión ubicado sobre México -parte del empuje frío-, aumentó la presión atmosférica en el Mar Caribe, generando un aumento importante en la intensidad el viento sobre el país. De acuerdo con los reportes aeronáuticos, las ráfagas de viento alcanzaron los $100 \mathrm{~km} / \mathrm{h}$ en los sectores montañosos, siendo el 3 de marzo el día más ventoso de este evento.

Evento 2, febrero, 2014

Se observó durante el periodo 12-21/02/2014 (Fig. 5), con una duración de 10 días, un promedio de $19.66{ }^{\circ} \mathrm{C}$ y un mínimo

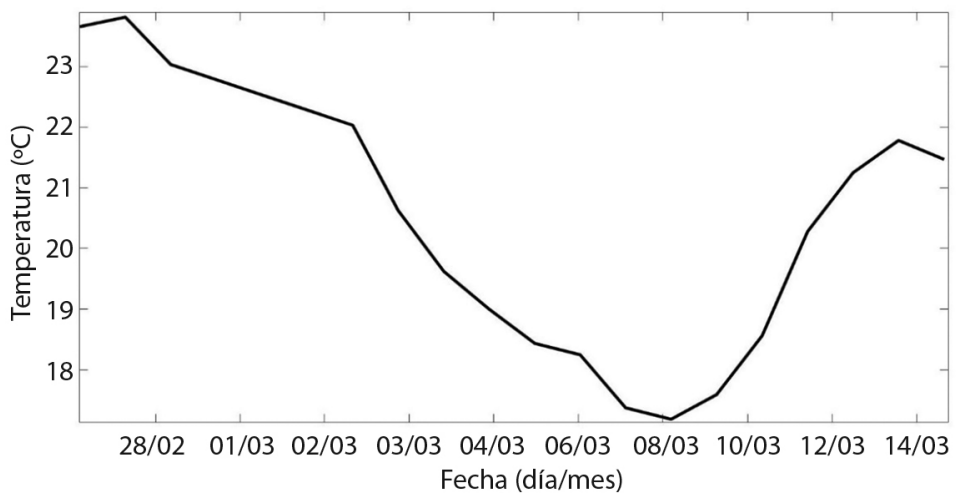

Fig. 4. Índice de Temperatura Subsuperficial del Mar (ITSbSM) durante el evento frío observado en el Golfo de Papagayo, 26/02-14/03/2013.

Fig. 4. Sea Subsurface Temperature Index (SSbTI) during the cold event observed in the Gulf of Papagayo, 26/02$14 / 03 / 2013$. 


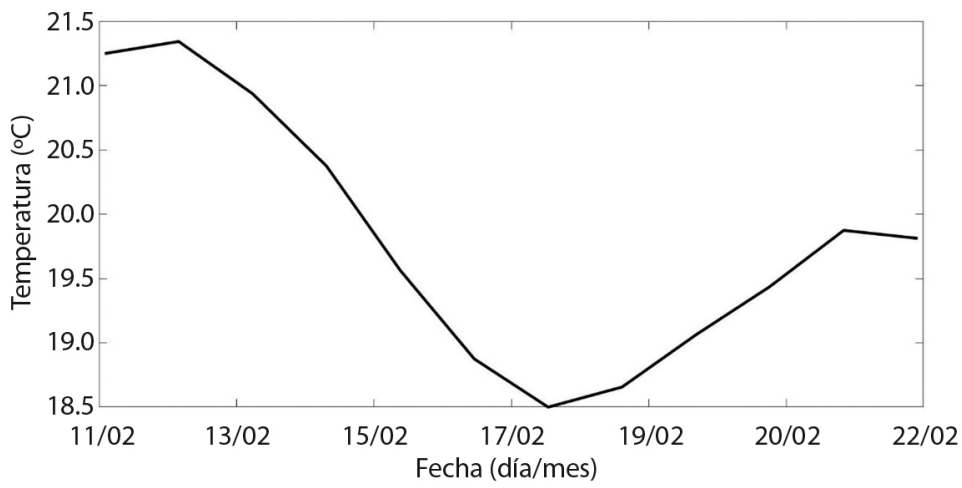

Fig. 5. Índice de Temperatura Subsuperficial del Mar (ITSbSM) durante el evento frío observado en el Golfo de Papagayo, $11-22 / 02 / 2014$

Fig. 5. Sea Subsurface Temperature Index (SSbTI) during the cold event observed in the Gulf of Papagayo, 11-22/02/2014.

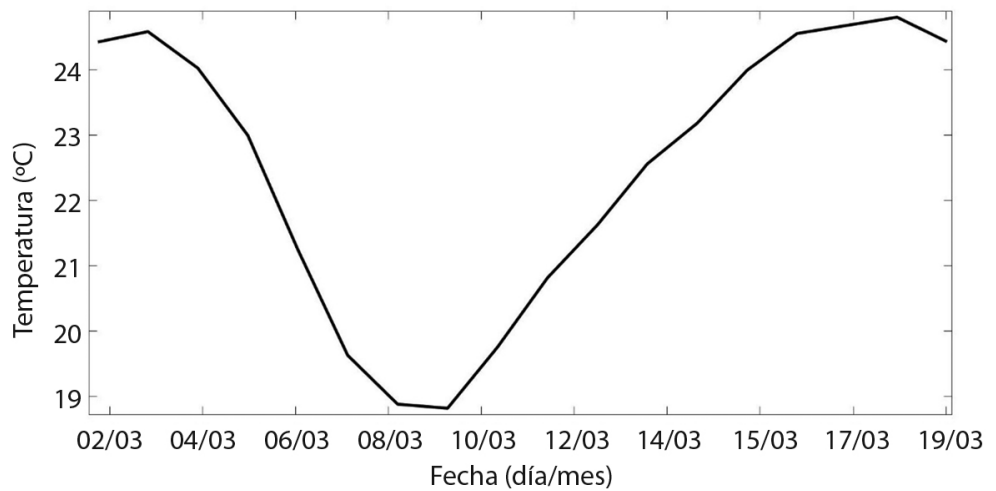

Fig. 6. Índice de Temperatura Subsuperficial del Mar (ITSbSM) durante el evento frío observado en el Golfo de Papagayo, 02-19/03/2019.

Fig. 6. Sea Subsurface Temperature Index (SSbTI) during the cold event observed in the Gulf of Papagayo, 02-19/03/2019.

de $18.50{ }^{\circ} \mathrm{C}$ el $17 / 02 / 2014\left(16^{\circ} \mathrm{C}\right.$ en el índice sin suavizar). Según Chinchilla (2014) hubo tres empujes fríos, 9-12 febrero, 13-14 febrero y 15-19 febrero, todos con fuertes vientos en Costa Rica, en Liberia el viento sostenido al medio día alcanzó hasta 23 nudos $(42.5 \mathrm{~km} / \mathrm{h})$ el 16 de febrero de 2014.

\section{Evento 3, marzo, 2019}

Este evento inició el 03/03/2019 y finalizó el 18/03/2019 (Fig. 6), con una duración de 16 días. Su promedio fue de $22.26{ }^{\circ} \mathrm{C}$, con un mínimo de $18.81{ }^{\circ} \mathrm{C}$ el $09 / 03 / 2019\left(16{ }^{\circ} \mathrm{C}\right.$ en el índice sin suavizar).
Según Morera (2019), hubo un empuje frío que ingresó al Mar Caribe a inicios de marzo. Un escenario ventoso predominó a lo largo del mes, dominando los vientos alisios en el Pacífico Norte, originados por los altos valores de la presión atmosférica sobre el Mar Caribe y Centroamérica. Los eventos de vientos más significativos fueron acentuados por la incursión de empujes fríos al norte de la región, el caso más relevante se manifestó en el periodo comprendido del 6 al 8 de marzo, y en Liberia con máximos a mediodía entre $35-50$ nudos $(64.82-92.6 \mathrm{~km} / \mathrm{h})$ y vientos superiores a 100 $\mathrm{km} / \mathrm{h}$ en el norte de Guanacaste. 


\section{Eventos Cálidos}

Evento 1, mayo, 2012

Este evento se observó en el periodo 07-18/05/2012 (Fig. 7), con una duración de 12 días. Su promedio fue de $30.18{ }^{\circ} \mathrm{C}$ con un máximo de $30.69{ }^{\circ} \mathrm{C}$ el $11 / 05 / 2012\left(31{ }^{\circ} \mathrm{C}\right.$ en el índice sin suavizar).

Según Solano (2012), en el Pacífico de Costa Rica, se observó la presencia de aguas cálidas, producto del paso hacia los polos de las ondas oceánicas Kelvin ecuatoriales, reflejadas desde la plataforma continental de Suramérica. Los indicadores oceánicos de mayo mostraron que el fenómeno ENOS se encontró en un estado de transición hacia El Niño.
Evento 2, abril-mayo, 2014

El inicio del evento fue el 29/04/2014 y su final el 10/05/2014 (Fig. 8), con una duración de 12 días, un promedio de $29.05^{\circ} \mathrm{C}$ y un máximo de $29.75{ }^{\circ} \mathrm{C}$ el $05 / 05 / 2014\left(30{ }^{\circ} \mathrm{C}\right.$ en el índice sin suavizar).

Según Morera (2014), en la mayor parte del país se manifestaron temperaturas más altas que el promedio mensual, las más altas se registraron en las estaciones del Pacífico, particularmente en Liberia que fue de $35.6{ }^{\circ} \mathrm{C}$, lo que representa $1.5^{\circ} \mathrm{C}$ más que su media climatológica. Los índices de la temperatura superficial del Océano Pacífico ecuatorial presentaron valores acordes con el desarrollo de un evento

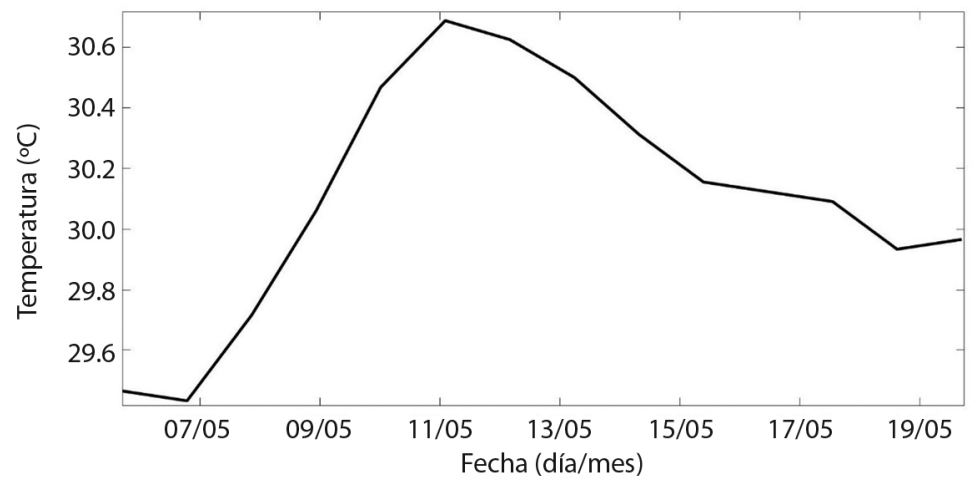

Fig. 7. Índice de Temperatura Subsuperficial del Mar (ITSbSM) durante el evento cálido observado en el Golfo de Papagayo, 06-19/05/2012.

Fig. 7. Sea Subsurface Temperature Index (SSbTI) during the warm event observed in the Gulf of Papagayo, 06-19/05/2012.

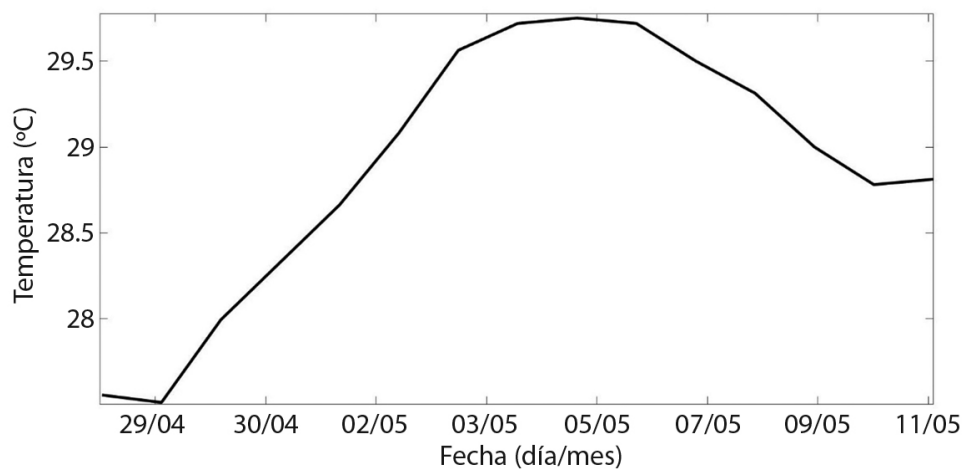

Fig. 8. Índice de Temperatura Subsuperficial del Mar (ITSbSM) durante el evento cálido observado en el Golfo de Papagayo, 28/04-11/05/2014.

Fig. 8. Sea Subsurface Temperature Index (SSbTI) during the warm event observed in the Gulf of Papagayo, 28/0411/05/2014. 


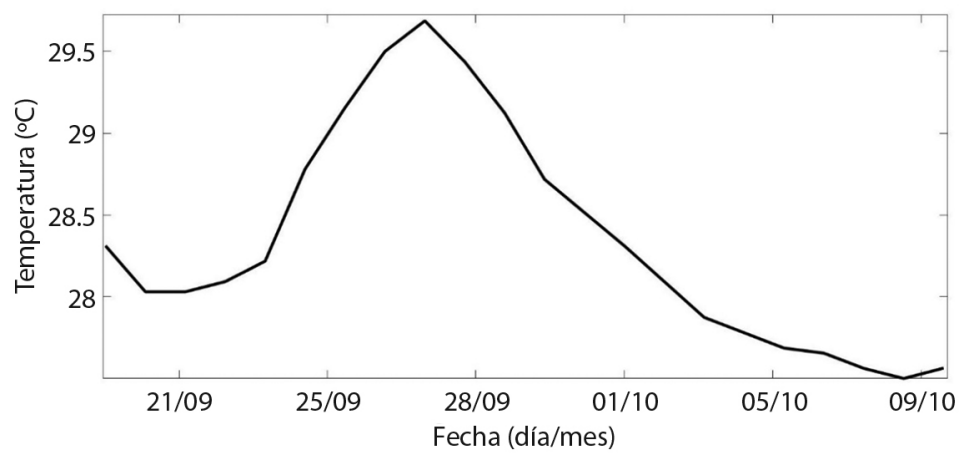

Fig. 9. Índice de Temperatura Subsuperficial del Mar (ITSbSM) durante el evento cálido observado en el Golfo de Papagayo, $19 / 09-09 / 10 / 2015$.

Fig. 9. Sea Subsurface Temperature Index (SSbTI) during the warm event observed in the Gulf of Papagayo, 19/09$09 / 10 / 2015$.

de El Niño, con anomalías positivas mayores a $+0.5^{\circ} \mathrm{C}$ en las temperaturas de la superficie del mar de las regiones centrales y orientales del océano Pacífico ecuatorial.

Evento 3, setiembre-octubre, 2015

Este evento inició el 20/09/2015 y finalizó el 08/10/2015 (Fig. 9), con una duración de 19 días. El promedio observado fue de $28.38{ }^{\circ} \mathrm{C}$, un máximo de $29.69{ }^{\circ} \mathrm{C}$ para el $27 / 09 / 2015$ (30 ${ }^{\circ} \mathrm{C}$ en el índice sin suavizar).

Según Naranjo (2015), Costa Rica estuvo bajo la influencia del fenómeno de El Niño. El fenómeno de El Niño tuvo un impacto en las temperaturas del aire, esto debido a la cercanía con el origen del fenómeno, lo cual facilitó el incremento en las mismas. Además, hubo un patrón deficitario en las precipitaciones. Alvarado (2015) agrega que la magnitud del fenómeno del Niño alcanzó en setiembre los niveles más altos desde el evento del Niño de 1997 y estuvo en el grupo de los tres más intensos desde 1950. La distribución horizontal de las anomalías de temperatura del mar mostró un calentamiento relativamente mayor en la parte más oriental del Pacífico ecuatorial (de hasta $3{ }^{\circ} \mathrm{C}$ ). En cuanto a la precipitación, en Liberia (Guanacaste) el déficit del 2015 fue el más alto de todo el registro histórico anterior, que data de 1937.

\section{DISCUSIÓN}

La iniciativa de Ciencia Ciudadana presentada en este estudio mostró ser muy útil para el monitoreo de la temperatura del mar en el Golfo de Papagayo, Guanacaste, Costa Rica. Lo anterior, debido a que los conjuntos de datos oceanográficos y programas de monitoreo a largo plazo en esta región son escasos (Alfaro \& Cortés, 2012; Alfaro et al., 2012; Mora-Escalante, Lizano, Alfaro, \& Rodríguez, 2020). Del registro de temperaturas obtenido por medio de esta iniciativa, se identificaron los tres eventos más fríos y más cálidos.

Los descensos durante los eventos fríos alcanzaron los $16-17^{\circ} \mathrm{C}$. Al igual que en Alfaro y Cortés (2012), todos los eventos fríos estuvieron asociados al paso de frentes fríos por el Mar Caribe (Zárate-Hernández, 2013), estos son un sistema sinóptico que refuerza la intensidad del viento alisio, con componente zonal del este y que provoca surgencia estacional, ya que las aguas superficiales son empujadas hacia afuera del golfo y estas son sustituidas por aguas más frías de capas más profundas (Alfaro \& Cortés, 2012). Estos eventos sucedieron durante el máximo invernal de la corriente en Chorro de Bajo Nivel del Caribe (Amador, 2008).

Los eventos cálidos presentaron temperaturas en sus máximos de $30-31{ }^{\circ} \mathrm{C}$. Los eventos 
estuvieron asociados al desarrollo y madurez de los eventos cálidos del ENOS tipo El Niño. El ENOS es un modulador importante de la variabilidad de la temperatura del mar en el Golfo de Papagayo, ya que los eventos El Niño están relacionados con anomalías positivas de la temperatura del mar en el Pacífico Tropical del Este (Alfaro et al., 2012; Alfaro \& Lizano, 2001). Las ondas Kelvin, atrapadas a la costa durante el desarrollo de estos eventos, posicionan la termoclina en niveles más profundos de la columna de agua (Solano, 2012). Las fuentes de variabilidad encontradas en este trabajo coinciden además con las reportadas recientemente por Alfaro y Cortés (2021).

Los resultados de este estudio indican que los guías de buceo pueden proporcionar datos sobre la temperatura del mar de calidad suficiente y con alta resolución temporal, en concordancia con lo mostrado por Weeser et al. (2018), quienes sugieren tres beneficios importantes de estas iniciativas de Ciencia Ciudadana: 1) los ciudadanos pueden apoyar de manera rentable el monitoreo, 2) las mediciones de fuentes colaborativas aumentan el acervo de datos en regiones poco estudiadas y 3) los ciudadanos pueden informar regularmente y con alta calidad la temperatura del agua en lugares remotos. Para aprovechar todo el potencial de la Ciencia Ciudadana en la cogeneración de conocimiento, estudios como los descritos en Alfaro y Cortés (2012) y Alfaro et al. (2012), podrían beneficiarse de más proyectos co-creados que establezcan fuertes lazos entre la investigación y la participación pública, mejorando así la sostenibilidad a largo plazo del monitoreo (Njue et al., 2019). Bonney, Phillips, Ballard y Enck (2015), encontraron pruebas sólidas de que los resultados científicos de la Ciencia Ciudadana están bien documentados. Hay evidencia de que estos proyectos logran ganancias de los participantes en el conocimiento sobre el problema y el proceso científico, aumentan la conciencia pública sobre la diversidad de la investigación científica y brindan un significado más profundo a las actividades de los participantes. Por último, mencionan que la Ciencia Ciudadana puede contribuir positivamente al bienestar social al influir en las preguntas que se están abordando y al dar voz a las personas en la toma de decisiones ambientales locales. La actividad de recolección de datos fue afectada por la pandemia del COVID-19 durante el año 2020, ya que la visitación turística disminuyó o fue nula, por lo que el principal reto es el de retomar la actividad de monitoreo en la región por parte de la empresa de buceo. También se debe motivar a las empresas a la divulgación de los resultados de este tipo de estudios en sus medios de comunicación, lo cual daría visibilidad tanto nacional como internacional a las empresas y motivaría a otras a desarrollar programas similares.

Declaración de ética: los autores declaran que todos están de acuerdo con esta publicación y que han hecho aportes que justifican su autoría; que no hay conflicto de interés de ningún tipo; y que han cumplido con todos los requisitos y procedimientos éticos y legales pertinentes. Todas las fuentes de financiamiento se detallan plena y claramente en la sección de agradecimientos. El respectivo documento legal firmado se encuentra en los archivos de la revista.

\section{AGRADECIMIENTOS}

Agradecemos a Francisca Blömeke por tener la brillante idea de recopilar los datos de buceo recreativo diariamente, con la única intención de agradar con excelente servicio, así como a Oliver Blömeke por el apoyo incondicional y confianza para el análisis de datos. Sin querer, su aventura como propietarios de la tienda Deep Blue Diving en Playa del Coco, Costa Rica, los hace merecedores de admiración y respeto en el campo de la Ciencia Ciudadana. A Mary McClave y Emanuel Vallejos de la tienda de buceo Deep Blue Diving, por su apoyo logístico a la actividad de monitoreo de Ciencia Ciudadana y colaborar con la recolección de los datos para este estudio. EA agradece el apoyo de los siguientes proyectos inscritos en la Vicerrectoría de Investigación de la Universidad de Costa Rica: B9-454 (VI-Grupos), 
EC-497 (FEES-CONARE), C0-610 (Fondo de Estímulo), A4-906 (PESCTMA-CIGEFI), C0-404 (PNUD), C0-074, A1-715, B0-810 y A5-037. También a la Escuelas de Física de la UCR por darle el tiempo de investigación para desarrollar este estudio, así como a los centros de investigación CIMAR y CIGEFI de la UCR por su apoyo logístico durante la recopilación y análisis de datos.

\section{RESUMEN}

Introducción: en el año 2011 y como una iniciativa de Ciencia Ciudadana, los propietarios de la tienda Deep Blue Diving Shop en Playa del Coco, Guanacaste, Costa Rica, iniciaron a recolectar diariamente los datos de temperatura en la columna de agua de mar durante sus actividades de buceo, como respuesta a la necesidad de informar a los turistas antes de viajar a Costa Rica para la selección del equipo correcto de SCUBA y así maximizar su experiencia en el Golfo de Papagayo. Esta recolección de datos se mantuvo constante hasta enero de 2020 por la pandemia del COVID-19.

Objetivo: identificar eventos fríos y cálidos de la temperatura sub-superficial del mar en el Golfo de Papagayo, Guanacaste, Costa Rica y su relación con fuentes de variabilidad climática conocidas como El Niño-Oscilación del Sur (ENOS) y sistemas sinópticos como las incursiones de frentes fríos en el Mar Caribe.

Métodos: se utilizaron datos de Temperatura Subsuperficial del Mar correspondientes a la temperatura más baja de la columna del agua de mar, a una profundidad aproximada de 25-35 m. Las observaciones se realizaron con una frecuencia diaria del 01/01/2011 al 31/01/2020.

Resultados: la iniciativa de Ciencia Ciudadana presentada en este estudio mostró ser muy útil para el monitoreo y caracterización de la temperatura del mar en el Golfo de Papagayo. La temperatura media fue de $25.7^{\circ} \mathrm{C}$. Se observaron temperaturas más frías en febrero-marzo, por debajo de $22.5{ }^{\circ} \mathrm{C}$ con un mínimo secundario en julio. Hubo dos máximos en mayo y agosto con temperaturas superiores a $27.4^{\circ} \mathrm{C}$. Los descensos durante los eventos fríos alcanzaros los $16-17^{\circ} \mathrm{C}$. Todos los eventos fríos estuvieron asociados al paso de frentes fríos por el Mar Caribe, debido a reforzamientos en intensidad del viento alisio, con componente zonal del este, que provoca surgencia estacional. Los eventos cálidos presentaron temperaturas en sus máximos de $30-31{ }^{\circ} \mathrm{C}$. Los eventos cálidos estuvieron asociados al desarrollo y madurez de los eventos cálidos del ENOS tipo El Niño. El ENOS es un modulador importante de la variabilidad de la temperatura del mar en el Golfo de Papagayo, ya que los eventos El Niño están relacionados con anomalías positivas de la temperatura del mar en el Pacífico Tropical del Este.
Conclusiones: la iniciativa de Ciencia Ciudadana presentada en este estudio resultó ser muy útil para monitorear la temperatura del mar en el Golfo de Papagayo. Los resultados de este estudio indican que los guías de buceo pueden aportar datos sobre la temperatura del mar de suficiente calidad y con alta resolución temporal. Los buzos pueden apoyar de manera rentable el monitoreo y la Ciencia Ciudadana puede contribuir positivamente al bienestar social al influir en las preguntas que se están abordando y al dar voz a las personas en la toma de decisiones ambientales locales. La información generada en este estudio regresa a los operadores de turismo y mejora la comprensión de la variabilidad observada en los datos recolectados por ellos, conocimiento que luego es transmitido a sus clientes para mejorar su experiencia.

Palabras clave: Ciencia Ciudadana; Golfo Papagayo; temperatura sub-superficial del mar; afloramiento-surgencia; América Central.

\section{REFERENCIAS}

Alfaro, E. J., \& Cortés, J. (2012). Atmospheric forcing of cool subsurface water events in Bahía Culebra, Gulf of Papagayo, Costa Rica. Revista de Biología Tropical, 60(Supplement 2), S173-S186.

Alfaro, E. J., \& Cortés, J. (2021). Climate forcing of cool and warm subsurface water events in Bahía Salinas, Costa Rica. Sometido a la Revista de Biología Tropical, 69(Supplement 2), S127-S141.

Alfaro, E. J., Cortés, J., Alvarado, J. J., Jiménez, C., León, A., Sánchez-Noguera, C., Nivia-Ruiz, J., \& Ruiz, E. (2012). Clima y temperatura superficial del mar en Bahía Culebra, Golfo de Papagayo, Costa Rica. Revista de Biología Tropical, 60(Suplemento 2), S159-S171.

Alfaro, E. J., \& Lizano, O. G. (2001). Algunas relaciones entre las zonas de surgencia del Pacífico Centroamericano y los Océanos Pacífico y Atlántico Tropical. Revista de Biología Tropical, 49(Suplemento 2), S185-S193.

Alvarado, L. (2015). Estado y pronóstico del fenómeno ENOS. Boletín Meteorológico Mensual. Instituto Meteorológico Nacional, San José, Costa Rica. https://www.imn.ac.cr/documents/10179/14641/ SETIEMBRE

Amador, J. A. (2008). The Intra-Americas Seas Low-Level Jet (IALLJ): Overview and Future Research. Annals of the New York Academy of Sciences, 1146(1), 153188. https://doi.org/10.1196/annals.1446.012

Amador, J. A., Alfaro, E. J., Lizano, O. G., \& Magaña, V.O. (2006). Atmospheric forcing in the Eastern Tropical Pacific: A review. Progress in Oceanography, 69, 101-142. 
Bonney, R., Phillips, T. B., Ballard, H. L., \& Enck, J. W. (2016). Can citizen science enhance public understanding of science? Public Understanding of Science, 25(1), 2-16. https://doi. org/10.1177/0963662515607406

Chinchilla, G. (2014). Resumen meteorológico: febrero de 2014. Boletín Meteorológico Mensual. Instituto Meteorológico Nacional, San José, Costa Rica. https://www.imn.ac.cr/documents/10179/14639/ FEBRERO

Jiménez, C., Cortés, J., León, A., \& Ruiz, E. (2001). Coral bleaching and mortality associated with the1997-98 El Niño in an upwelling environment in the Eastern Pacific (Gulf of Papagayo, Costa Rica). Bulletin of Marine Science, 69, 151-169.

Mora-Escalante, R. E., Lizano, O. G., Alfaro, E. J., \& Rodríguez, A. (2020). Distribución de temperatura y salinidad en campañas oceanográficas recientes en el Pacífico Tropical Oriental de Costa Rica. Revista de Biología Tropical, 68(Suplemento 1), S177-S197.

Morera, R. (2014). Resumen meteorológico y condición actual del fenómeno ENOS: mayo de 2014. Boletín Meteorológico Mensual. Instituto Meteorológico Nacional, San José, Costa Rica. https://www.imn. ac.cr/documents/10179/14639/MAYO

Morera, R. (2019). Resumen meteorológico: marzo de 2019. Boletín Meteorológico Mensual. Instituto Meteorológico Nacional, San José, Costa Rica. https://www.imn.ac.cr/documents/10179/474355/ MARZO

Naranjo, D. (2013). Resumen meteorológico: marzo de 2013. Boletín Meteorológico Mensual. Instituto Meteorológico Nacional, San José, Costa Rica. https://www.imn.ac.cr/documents/10179/14637/ MARZO
Naranjo, D. (2015). Resumen meteorológico: setiembre de 2015. Boletín Meteorológico Mensual. Instituto Meteorológico Nacional, San José, Costa Rica. https://www.imn.ac.cr/documents/10179/14641/ SETIEMBRE

Njue, N., Stenfert-Kroese, J., Gräf, J., Jacobs, S. R., Weeser, B., Breuer, L., \& Rufino, M. C. (2019). Citizen science in hydrological monitoring and ecosystem services management: State of the art and future prospects. Science of the Total Environment, 693, 133531. https://doi.org/10.1016/j.scitotenv.2019.07.337.

Shahady, T., \& Boniface, H. (2018). Water quality management through community engagement in Costa Rica. Journal of Environmental Studies and Sciences, 8, 488-502. https://doi.org/10.1007/ s13412-018-0504-7.

Solano, E. (2012). Estado del fenómeno ENOS, transición al Niño: mayo de 2012. Boletín Meteorológico Mensual. Instituto Meteorológico Nacional, San José, Costa Rica. https://www.imn. ac.cr/documents/10179/14635/MAYO

Soley, F. J. (1994). Suavizamiento de series cronológicas geofísicas con ruido blanco y rojo aditivo. Revista Geofísica, 41, 33-58.

Weeser, B., Stenfert-Kroese, J., Jacobs, S. R., Njue, N., Kemboi, Z., Ran, A., Rufino, M. C., \& Breuer, L. (2018). Citizen science pioneers in Kenya - A crowdsourced approach for hydrological monitoring. Science of the Total Environment, 631-632, 1590-1599. https://doi.org/10.1016/j.scitotenv.2018.03.130.

Zárate-Hernández, E. (2013). Climatología de masas invernales de aire frío que alcanzan Centroamérica y el Caribe y su relación con algunos índices Árticos. Tópicos Meteorológicos y Oceanográficos, 12(1), 35-55. 